\title{
Personalized Predictive Models for Identifying Clinical Deterioration Using LSTM in Emergency Departments
}

\author{
Amin NAEMI ${ }^{1, \mathrm{a}}$,Thomas SCHMIDT ${ }^{\mathrm{a}}$, \\ Marjan MANSOURVAR ${ }^{\mathrm{a}}$ and Uffe Kock WIIL ${ }^{\mathrm{a}}$ \\ ${ }^{a} S D U$ Health Informatics and Technology, The Maersk Mc-Kinney Moeller Institute, \\ University of Southern Denmark, Denmark
}

\begin{abstract}
Early detection of deterioration at hospitals could be beneficial in terms of reducing mortality and morbidity rates and costs. In this paper, we present a model based on Long Short-Term Memory (LSTM) neural network used in deep learning to predict the illness severity of patients in advance. Hence, by predicting health severity, this model can be used to identify deteriorating patients. Our proposed model utilizes continuous monitored vital signs, including heart rate, respiratory rate, oxygen saturation, and blood pressure automatically collected from patients during hospitalization. In this study, a short-time prediction using a sliding window approach is applied. The performance of the proposed model was compared with the Multi-Layer Perceptron (MLP) neural network, a feedforward class of neural network, based on $\mathrm{R}^{2}$ score and Root Mean Square Error (RMSE) metrics. The results showed that the LSTM has a better performance and could predict the illness severity of patients more accurately.
\end{abstract}

Keywords. clinical deterioration, machine learning algorithms, time series, health informatics, LSTM, recurrent neural network, emergency department.

\section{Introduction}

Research has demonstrated that about 31 percent of acutely admitted patients, who seem normal upon arrival deteriorate during their stay, which could lead to an increase in mortality and morbidity rate [1]. The probability of deterioration and adverse events such as unexpected transfer to the intensive care unit can be reduced by using consistent and rigorous methods of vital signs monitoring and applying deterioration prediction models $[2,3]$. Therefore, many departments utilize expensive patient monitoring equipment. However, most of the automatic measurements recorded by these devices are never used in clinical decision making, due to the large amount of information that is timeconsuming and difficult for clinicians to process. Consequently, potentially useful information for identifying impending deterioration is neglected. Through monitoring of patients and conversations with clinicians, it is evident that the health condition of a patient is difficult to quantify [4]. However, dynamic changes in patients' vital signs can be used to detect those who are at risk of adverse events and deterioration. Several Machine Learning (ML) algorithms such as Neural Network (NN), Support Vector

\footnotetext{
${ }^{1}$ Corresponding Author, Amin Naemi, E-mail: amin@mmmi.sdu.dk.
} 
Machine (SVM), K-Nearest Neighbors (K-NN), Gaussian Process (GP) have been utilized to predict patient severity, instability, and clinical deterioration [5]. Most of these studies showed that ML techniques could recognize complex patterns and predict the health condition of patients and clinical deterioration more precisely than traditional approaches. However, a majority of these studies neglected previous monitored information, long dependencies, and dynamic changes in illness severity of patients; however, the current health status of patients depends on their previous health conditions. One of the appropriate algorithms that can address this limitation and detect temporal dependencies and dynamic changes in a time series is Long Short-Term Memory (LSTM) neural network. The main idea behind using LSTM is to investigate whether there is a temporal and long-term dependency in a time series. LSTM has been used in Emergency Departments (ED) such as prediction of admission [6], and pain classification [7]. Moreover, recent studies in medicine indicate that the population of patients is heterogeneous, i.e., every patient has his/her unique characteristics, and it is necessary to have targeted, patient-specific predictions and treatments [8]. Nevertheless, most of the studies have proposed a single model for the whole population. Hence, it is important to examine the impact of building personalized models on the quality of model performance. Therefore, in this paper, a LTSM neural network model is proposed to predict clinical deterioration and patient illness severity on the individual level at ED. This model utilizes continuous monitored vital signs and considers the previous health conditions and characteristics of each patient to predict the future illness severity of them. This enables the clinicians to identify patients who are at risk of deterioration and helps them to manage their medical resources and attention more effectively.

\section{Materials}

\subsection{Data Acquisition}

Vital signs of patients, including Heart Rate (HR), Respiratory Rate (RR), Arterial Blood Oxygen Saturation (SpO2), and systolic Blood Pressure (BP) of all patients admitted to the EDs of Odense University Hospital (OUH), and Hospital of South Western Jutland (HSWJ) between 2018 and 2019 were stored in the database. This data was gathered from the HL7 interface of Philips IntelliVue patient monitors and registered in 60-second intervals. Moreover, other information such as age, gender, admission date, length of stay, and clinical notes was stored in the dataset. Vital signs registration was authorized by the Danish Data Protection Agency under journal nr. 17/14630 and registered at ClinicalTrials.gov with number: NCT03375658. The data was stored in a database with restricted access according to Danish legislation on privacy concerns and analyzed by Python 3.7.7. Description of the data is shown in Table 1.

Table 1. Data description

\begin{tabular}{|c|c|c|}
\hline & OUH & HSWJ \\
\hline Patients, $n$ & $31,234$ (male: 16,271$)$ & 21,421 (male: 12,054 ) \\
\hline Age, Median (years) & 65 (IQR: $51-80)$ & 66 (IQR: $54-81)$ \\
\hline HR, Median (minute (1) $^{-1}$ & 83 (IQR: $74-111$ ) & 81 (IQR: $70-106)$ \\
\hline RR, Median (minute ${ }^{-1}$ ) & 16 (IQR: $14-22$ ) & 18 (IQR: $14-23$ ) \\
\hline SpO2, Median (\%) & 97 (IQR: 92 - 99) & 97 (IQR: $93-99)$ \\
\hline BP, Median (mmHg) & 126 (IQR: $119-131)$ & 129 (IQR: 118 - 136) \\
\hline Length of Monitoring, Median (minute) & 181 (IQR: $98-721)$ & $523(271-1176)$ \\
\hline Total Vital signs, $n$ & $2,488,292$ & $5,619,021$ \\
\hline
\end{tabular}




\subsection{Data Preprocessing}

In the preprocessing phase, patients under 18 years old and patients not monitored during their admission, were removed from the dataset to prepare the data for the model development phase. To handle missing values in vital signs, Moving Average (MA) technique with a window size of 10 minutes was used.

\section{Methods}

\subsection{Scoring System}

The primary goal of scoring systems is to stratify patients into different categories based on their health conditions and help clinicians to identify seriously ill patients [9]. In this study, Adaptive Process Triage (ADAPT) was used to assign patients to different categories based on their vital signs. ADAPT has been utilized in several Danish hospitals and could be used for scoring the severity of vital signs according to the ABCDE-principle [9]. Table 2 shows how the ADAPT triage categories are computed based on vital signs. For each patient, based on his/her vital signs over time, a sequence of severity scores is produced. ADAPT has four categories including Red, Orange, Yellow, and Green, where Red indicates more severe circumstances that require a higher priority and Green corresponds to less urgent situations that can be handled with a lower priority.

Table 2. ADAPT triage model

\begin{tabular}{|c|c|c|c|c|}
\hline & $\begin{array}{l}\text { Red (1) } \\
\text { Resuscitation } \\
0 \text { min } \\
\end{array}$ & $\begin{array}{c}\text { Orange (2) } \\
\text { Urgent } \\
15 \mathrm{~min} \\
\end{array}$ & $\begin{array}{l}\text { Yellow (3) } \\
\text { Less urgent } \\
60 \mathrm{~min}\end{array}$ & $\begin{array}{c}\text { Green (4) } \\
\text { Not urgent } \\
180 \mathrm{~min} \\
\end{array}$ \\
\hline Airways & $\begin{array}{l}\text { Obstructed } \\
\text { airway stridor }\end{array}$ & $\begin{array}{l}\text { threatened } \\
\text { airway }\end{array}$ & & \\
\hline Breathing & $\begin{array}{l}\mathrm{SpO}_{2}<80 \% \\
8>\mathrm{RR}>35\end{array}$ & $\begin{array}{l}80<\mathrm{SpO}_{2}<89 \\
31<\mathrm{RR}<35\end{array}$ & $\begin{array}{l}90<\mathrm{SpO}_{2}<94 \\
26<\mathrm{RR}<30\end{array}$ & $\begin{array}{l}\mathrm{SpO}_{2} \geq 95 \\
8<\mathrm{RR}<25\end{array}$ \\
\hline Circulation & $\begin{array}{l}\mathrm{HR}>130 \\
\mathrm{BP}_{\text {sys }}<80\end{array}$ & $\begin{array}{l}121<\mathrm{HR}<130 \\
\mathrm{HR}<40 \\
80<\mathrm{BP}_{\mathrm{sys}}<89\end{array}$ & $\begin{array}{l}111<\mathrm{HR}<120 \\
40<\mathrm{HR}<49\end{array}$ & $50<\mathrm{HR}<110$ \\
\hline Disability & $\mathrm{GCS} \leq 8$ & $9<\mathrm{GCS}<13$ & $\mathrm{GCS}=14$ & $\mathrm{GCS}=15$ \\
\hline Exposure & & $\begin{array}{l}\mathrm{T}_{\mathrm{p}}>40 \\
\mathrm{~T}_{\mathrm{p}}<32\end{array}$ & $\begin{array}{l}38.1<\mathrm{T}_{\mathrm{p}}<40 \\
32<\mathrm{T}_{\mathrm{p}}<34\end{array}$ & $34.1<\mathrm{T}_{\mathrm{p}}<38$ \\
\hline
\end{tabular}

\subsection{Model Development}

In this paper, two different types of neural networks, including Multi-Layer Perceptron (MLP) and LSTM were utilized to predict patients' severity. MLP is a well-known class of feedforward neural network while LSTM is a special kind of Recurrent Neural Networks (RNN) which has internal memory to process any arbitrary flow of inputs. In LSTM, usual hidden layers are substituted by LSTM cells. These cells consist of different gates, including input gate, output gate, and forget gate. 4-fold cross-validation was used to have a robust model, where the three folds were used to train the model each time, and the fourth fold was used as the validation set. Grid search was used to find the optimal hyperparameters and best models on the validation set were used as the final models to apply on the test data. Moreover, the sliding window approach was used for short-term prediction. This technique enables us to follow the dynamic and sudden changes in patients' conditions time series. The window size was set to 90 minutes, and the step size which indicates the number of predicted samples was set to 30 minutes. In other words, 
the severity of a patient for the next 30 minutes was predicted based on his/her severity in the last 90 minutes. The training process was interrupted for both models after 1000 epochs to estimate the generalization error on the validation data. Moreover, the training process was stopped whenever the generalization error was higher than the error of previous epoch. The loss function was Mean Square Error (MSE), and the optimization algorithm to update networks weights was Adaptive Moment Estimation (Adam) [10] which was designed specifically for training deep neural networks. This algorithm has some advantages, including easy implementation, accelerate training, and suitable for non-stationary or noisy objectives.

\section{Results and Discussion}

2000 patients with at least 10 -hour monitoring time were selected randomly to evaluate the performance of LSTM. 75\% of this data was used as a training set and the rest was used for testing. The training set was used for training and validation process based on cross-validation. The hyperparameters of LSTM and MLP are shown in Table 3.

Table 3. Hyperparameters of models

\begin{tabular}{ccccc}
\hline & Hidden Layers & $\begin{array}{c}\text { Hidden Layers } \\
\text { Neurons }\end{array}$ & $\begin{array}{c}\text { Number } \\
\text { of Epochs }\end{array}$ & $\begin{array}{c}\text { Optimization } \\
\text { Algorithm }\end{array}$ \\
\hline LSTM & 2 & 4,4 (LSTM cells) & 1000 & Adam \\
MLP & 2 & 8,4 & 1000 & Adam \\
\hline
\end{tabular}

Models were tested on the $25 \%$ of the data, selected as test data. $R^{2}$ score and Root Mean Square Error (RMSE) were used to evaluate the performance of models. The $R^{2}$ scores used in time series analysis is a value between 0 and 1, indicates how much of variance in a given time series can be explained by a model. A closer $R^{2}$ score to 1 means that the model works better. The average $R^{2}$ scores and RMSE for 500 patients (test data) are shown in Table 4. A patient was randomly selected to investigate the prediction accuracy of models, and the patient's severity for a sample period of 200 minutes was predicted, as shown in Figure 1.

Table 4. Performance of models

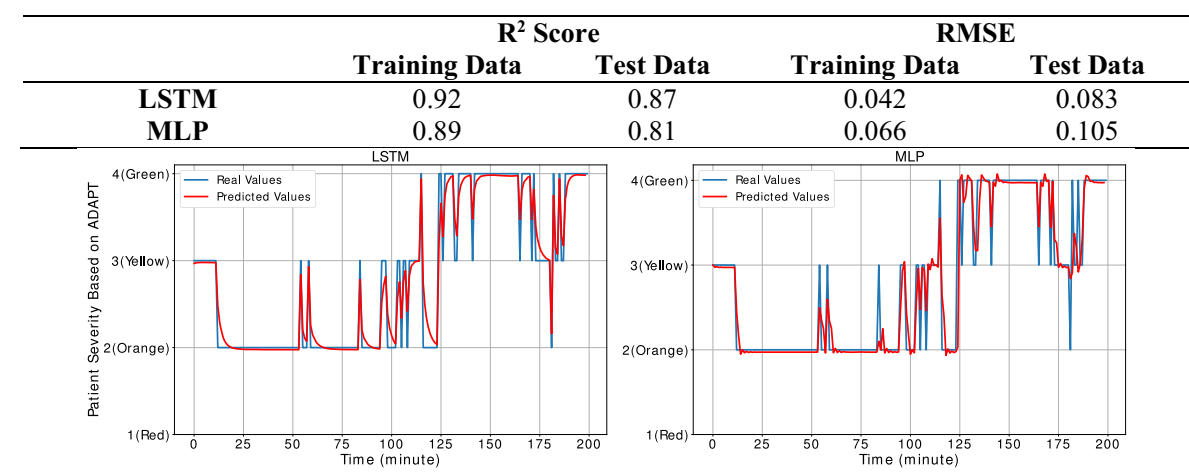

Figure 1. Prediction of a patient's illness severity using LSTM and MLP over a sample period.

Based on Table 4, the prediction performance of LSTM was better for both evaluation metrics. It means that there are meaningful information and long dependencies in patients' severity trajectories that must be taken into account for the prediction of future severity of patients. Moreover, based on Figure. 1, LSTM followed 
the real trajectory (blue time series) better than MLP, especially in sudden changes and high fluctuation situations. It shows that deep learning models such as LSTM have great capability in detecting dynamic changes of chaotic time series.

\section{Conclusion}

In this paper, we proposed a model based on the LSTM neural network to predict patients' illness severity, which is an index to quantify clinical deterioration, during hospitalization at EDs. The ADAPT triage system was used to calculate the severity of patients based on their vital signs. Our proposed method predicts patients' severity of illness individually using continuous monitored vital signs, automatically collected during treatment. This means that a separate model is developed for each patient trained based on patient characteristics and his/her previous recorded vital signs. Moreover, sliding window technique was applied to detect and follow the dynamic and sudden changes in patients' health conditions. The performance of the proposed model for a selected representative population of patients was investigated, and the results showed that the performance of LSTM, a recurrent deep neural network, is approximately $6 \%$ and $21 \%$ higher compared to the feedforward class of neural networks (MLP) in terms of RMSE and $\mathrm{R}^{2}$ score, respectively. This highlights the importance of considering long dependencies in patients' vital signs and previous health conditions for prediction of their severity. It also demonstrates the capability of deep neural networks such as LSTM to detect hidden and complex dynamics of time series. Future studies will focus on the prediction of each vital sign trajectories independently and developing ML models to predict clinical deterioration based on vital signs trajectories.

\section{References}

[1] Henriksen DP, Brabrand M, Lassen AT. Prognosis and risk factors for deterioration in patients admitted to a medical emergency department. PLoS One. 2014;9(4):e94649.

[2] Mansourvar M, Andersen-Ranberg K, Nøhr C, Wiil UK. A Predictive Model for Acute Admission in Aged Population. In: MIE. 2018. p. 96-100.

[3] Churpek MM, Adhikari R, Edelson DP. The value of vital sign trends for detecting clinical deterioration on the wards. Resuscitation. 2016;102:1-5.

[4] Schmidt T, Wiil UK. Identifying patients at risk of deterioration in the Joint Emergency Department. Cognition, Technology \& Work. 2015;17(4):529-45.

[5] Churpek MM, Yuen TC, Winslow C, Meltzer DO, Kattan MW, Edelson DP. Multicenter comparison of machine learning methods and conventional regression for predicting clinical deterioration on the wards. Critical Care Medicine. 2016;44(2):368.

[6] Kadri F, Baraoui M, Nouaouri I. An LSTM-based Deep Learning Approach with Application to Predicting Hospital Emergency Department Admissions. In: 2019 International Conference on Industrial Engineering and Systems Management (IESM). IEEE; 2019. p. 1-6.

[7] Tsai F-S, Weng Y-M, Ng C-J, Lee C-C. Embedding stacked bottleneck vocal features in a LSTM architecture for automatic pain level classification during emergency triage. In: 2017 Seventh International Conference on Affective Computing and Intelligent Interaction (ACII). IEEE; 2017. p. 313-8.

[8] Ng K, Sun J, Hu J, Wang F. Personalized predictive modeling and risk factor identification using patient similarity. AMIA Summits Translational Science Proceedings. 2015;2015:132.

[9] Nordberg M, Lethvall S, Castrén M. The validity of the triage system ADAPT. Scandinavian Journal of Trauma Resuscitation Emergency Medicine. 2010;18(S1):P36.

[10] Kingma DP, Ba J. Adam: A method for stochastic optimization. arXiv Preprint arXiv14126980. 2014. 